\title{
Linx
}

Revue des linguistes de l'université Paris X Nanterre

$80 \mid 2020$

L'héritage de Jean Dubois et Françoise Dubois-Charlier

\section{Présentation du numéro}

\section{Laetitia Leonarduzzi}

\section{(2) OpenEdition}

Journals

Édition électronique

URL : http://journals.openedition.org/linx/5336

DOI : 10.4000/linx.5336

ISSN : 2118-9692

Éditeur

Presses universitaires de Paris Nanterre

Référence électronique

Laetitia Leonarduzzi, «Présentation du numéro », Linx [En ligne], 80 | 2020, mis en ligne le 10 juillet 2020, consulté le 25 septembre 2020. URL : http://journals.openedition.org/linx/5336 ; DOI : https:// doi.org/10.4000/linx.5336

Ce document a été généré automatiquement le 25 septembre 2020.

Département de Sciences du langage, Université Paris Ouest 


\section{Présentation du numéro}

\section{Laetitia Leonarduzzi}

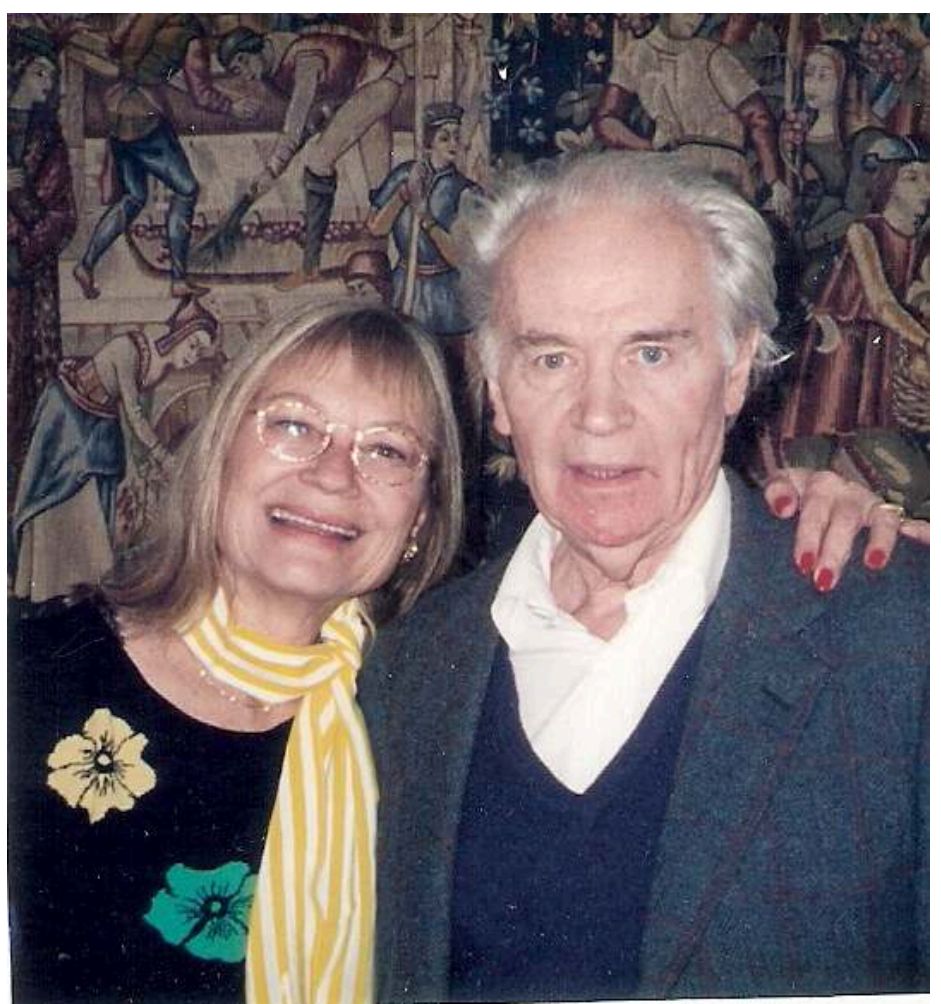

Françoise Dubois-Charlier et Jean Dubois vers 2003

L'union, dans la vie privée comme dans la vie professionnelle, de Jean Dubois (1920-2015) et Françoise Dubois-Charlier (1941-2016) a débouché sur une œuvre scientifique collaborative d'une qualité et d'une puissance exceptionnelles. Ce numéro ${ }^{1}$ tente de rendre compte de leurs travaux de la façon la plus fidèle et la plus extensive possible ${ }^{2}$. Il a ainsi l'ambition d'être moins une publication d'actes de colloque ou un recueil d'articles sur une thématique, qu'un monument modeste à la gloire de ces deux linguistes dont l'influence est considérable dans l'histoire de la linguistique française et de la linguistique anglaise en France, mais aussi par-delà nos frontières, comme en 
témoignent certains articles de ce numéro ainsi que plusieurs communications au colloque d'Aix-en-Provence qui l'a précédé3.

Plusieurs numéros de revue ont déjà été consacrés aux travaux de Jean Dubois et Françoise Dubois-Charlier : nous renvoyons le lecteur au numéro 153 de la revue Langue française (2007/1) ainsi qu'au numéro 179-180 de la revue Langages (2010). Précédemment, la revue LINX avait également consacré, en 1996, le numéro 34-35 aux travaux du seul Jean Dubois. A cela s'ajoute l'interview de Jean Dubois par Jean-Claude Chevalier et Pierre Encrevé que l'on trouve dans Combats pour la linguistique (2006, ENS Editions, pp. 145-166).

\section{Bref aperçu des travaux de Jean Dubois et Françoise Dubois-Charlier}

3 La puissance de travail des deux auteurs fut considérable : 17 livres et 7 articles publiés par le couple en linguistique française; Jean Dubois est aussi l'auteur de 16 autres ouvrages et 46 articles de linguistique française, seul ou en collaboration avec d'autres auteurs que Françoise Dubois-Charlier. Cette dernière a publié dans le domaine du français mais aussi de l'anglais (seule ou en collaboration) pas moins d'une vingtaine d'ouvrages (grammaires, dictionnaires, manuels de tous niveaux) et articles de linguistique anglaise (en particulier en syntaxe), auxquels s'ajoutent plusieurs ouvrages de linguistique générale et d'introduction à la linguistique (en collaboration), traduits en plusieurs langues. D'autre part, Jean Dubois et Françoise Dubois-Charlier se sont intéressés à la neurolinguistique : un livre et 25 articles (en collaboration) pour Jean Dubois, et un ouvrage et 5 articles pour Françoise Dubois-Charlier. Enfin, ils ont produit deux dictionnaires informatisés, Les Verbes français (LVF) et le Dictionnaire Electronique des Mots (DEM), qui sont d'une extension et d'une originalité exceptionnelles.

4 Jean Dubois et Françoise Dubois-Charlier ont largement contribué, dans les années 1960-1970, à l'introduction en France de la linguistique américaine. Le fait que Jean Dubois a été l'un des principaux responsables des dictionnaires et revues au sein des Editions Larousse a facilité son impact sur la vie intellectuelle des années 1960-1970. Les revues Langages et Langue Française sont lancées respectivement en 1966 et en 1968 dans le sillage de la mouvance structuraliste. Françoise Dubois-Charlier a elle-même contribué de son côté à l'essor de la grammaire générative et transformationnelle, puis de la sémantique générative et de la grammaire des cas à travers plusieurs ouvrages sur l'anglais ou le français et nombre de traductions de livres et d'articles. Jean Dubois et Françoise Dubois-Charlier ont par ailleurs été directeurs de publication pour la série Enseignement et Pédagogie chez Larousse.

5 Dans les années 1980, Jean Dubois et Françoise Dubois-Charlier se rapprochent de Maurice Gross, qui élabore avec son équipe, le LADL, un «lexique-grammaire » du français. Cette rencontre détermine chez eux un changement d'orientation théorique : leurs derniers travaux, principalement leurs dictionnaires informatisés, relèvent plus nettement qu'auparavant de la tradition des grammaires distributionnelles et transformationnelles initiée par Zellig Harris. Les dernières années de la collaboration de Jean Dubois et Françoise Dubois-Charlier furent une période d'intense activité, jusqu'à la mort de Jean Dubois en avril 2015. Le LVF et le DEM sont déjà accessibles sous leurs diverses versions sur le site de l'Université de Montréal et sur le site de l'équipe de 
recherche MoDyCo (CNRS et Université de Paris Nanterre). Ils ont déjà connu une assez large diffusion auprès des universitaires et de leurs étudiants. Certains des derniers travaux de Dubois et Dubois-Charlier restent inédits mais seront prochainement en libre accès sur le site du MoDyCo (il s'agit notamment de Locutions en français, Dictionnaire des suffixes, et Composition et préfixation). Dans les années 2000 les deux auteurs, en collaboration avec Paul Sabatier, ont révisé LVF et poursuivi l'élaboration de leur colossal Dictionnaire Electronique des Mots (DEM), qui restera inachevé.

\section{Présentation des articles}

Le numéro est divisé en quatre sections. La première section est consacrée aux travaux de Jean Dubois et la deuxième section se concentre sur la collaboration de Jean Dubois et Françoise Dubois-Charlier. Dans la troisième section, c'est Françoise Dubois-Charlier qui est à l'honneur, avec des textes qui concernent ses travaux ou qui, signés par d'anciens étudiants ou collègues, évoquent son rayonnement. Dans la quatrième section, on trouvera des témoignages de personnes qui ont connu Jean Dubois et Françoise Dubois-Charlier, ainsi que les bibliographies de ces derniers.

7 Dans la première section, consacrée plus spécifiquement à Jean Dubois, le lecteur trouvera d'abord un hommage rendu à ce dernier par Danielle Leeman, qui l'a beaucoup fréquenté tant dans la vie professionnelle que dans la vie privée.

Suivront deux articles qui rendent compte principalement des travaux de Jean Dubois. Dans une perspective d'histoire des idées linguistiques, l'article de Jacqueline Léon situe le travail de Jean Dubois dans son contexte historique et examine le rôle de passeur de ce dernier dans l'introduction de la linguistique américaine en France. L'article de Gaston Gross évoque les manuels de grammaire structurale de Jean Dubois (1965, 1967, 1969) ainsi que son ouvrage en collaboration avec Françoise DuboisCharlier Eléments de linguistique française : syntaxe (1970). Deux autres articles traitent de questions méthodologiques et théoriques. L'article de Lucien Kupferman revient sur les propriétés d'indissociabilité (paramètre 1) et de non commutabilité (paramètre 2) des locutions prépositionnelles (LP), plus particulièrement spatiales, du type à $(\mathrm{NP}+\mathrm{DP})_{s p}{ }^{1}$ de $(\mathrm{NP}+\mathrm{DP})^{2}$ et prend appui sur la LP à l'abri de pour montrer de façon nuancée le caractère non-référentiel de DP1 dans ces locutions et ainsi énoncer un nouveau paramètre (paramètre 3) qui englobe et explique les deux premiers tout en affinant la description et en donnant des résultats plus précis. L'article de Danielle Leeman et Max Silberztein, enfin, constitue une analyse distributionnelle de certains termes relevant du domaine de l'habitation dans deux types de discours différents ; cet article entend montrer que le lexique d'une langue est un reflet des représentations sociales ; la démonstration s'appuie en partie sur une grammaire implémentée avec le logiciel NooJ.

9 La deuxième section du numéro, qui examine les travaux effectués en collaboration par Jean Dubois et Françoise Dubois-Charlier, notamment leurs dictionnaires informatisés, regroupe six articles. Deux d'entre eux constituent une réflexion sur les méthodologies utilisées pour la constitution de dictionnaires. L'article de Maria-Luisa FernandezEchevarria s'intéresse à la conception de plateformes électroniques et au fonctionnement des moteurs de recherche dans les dictionnaires électroniques, d'une part en présentant les ressemblances entre les Classes génériques de Jean Dubois et Françoise Dubois-Charlier et les Idées clés d'un dictionnaire de locutions espagnol 
précurseur du moteur de recherche de proverbes en ligne de l'Institut Cervantes, d'autre part en mettant en avant les affinités entre les relations actionnelles de la Frame Based Theory et les schèmes syntaxiques dans Les verbes français. Adoptant une approche métalexicographique, l'article d'Antoinette Balibar-Mrabti s'interroge, dans une perspective historique, sur les savoir-faire et procédés qui président à la sélection des "phrases simples» qui illustrent les entrées verbales du LVF, plus particulièrement dans le cas des emplois figurés.

Deux autres articles illustrent également le type de recherches qui peuvent être effectuées à partir de LVF et du DEM. Elisabeth Godbert montre comment les informations sémantiques peuvent être extraites du DEM et de LVF en vue d'une détection automatique des relations de co-référence intra- et inter-phrases dans une perspective de traitement automatique du langage (TAL). L'article de Max Silberztein montre quant à lui comment les ressources linguistiques des dictionnaires DEM et LVF peuvent être exploitées pour traiter automatiquement les corpus utilisés dans le domaine des Humanités Numériques, en traitant les unités de sens plutôt que les formes graphiques, ce qui permet de produire des analyses statistiques plus précises et plus fiables.

11 Deux articles, enfin, commentent les travaux en collaboration de Jean Dubois et Françoise Dubois-Charlier. Celui de Guy Lapalme et Denis Le Pesant, revient assez brièvement sur LVF et développe une analyse de l'énorme base de données lexicales informatisées qu'est le DEM; l'article présente en outre une nouvelle version, combinée, de l'outil de consultation de LVF et du DEM disponible sur un site de l'Université de Montréal. L'article de Denis Le Pesant est un essai de récapitulation de l'ensemble de la carrière des deux linguistes.

12 La troisième section du numéro regroupe neuf articles sur l'héritage intellectuel de Françoise Dubois-Charlier. Trois d'entre eux se situent dans une perspective historique. Les deux premiers concernent des éléments de la carrière de Françoise Dubois-Charlier. Le premier, écrit par Jacques François, retrace les débuts de la revue Langages (créée par Jean Dubois en 1966) pour évaluer le rôle de F. Dubois-Charlier dans le développement en France des théories syntaxiques et sémantiques américaines ainsi que de la neurolinguistique. Le second, rédigé par Jacqueline Guéron, resitue l'œuvre de Françoise Dubois-Charlier dans un contexte d'histoire de la linguistique en développant en particulier le domaine de la grammaire générative et transformationnelle. Dans une perspective historique également, un troisième article, celui de Jean-Charles Khalifa, dresse un panorama de l'évolution de l'analyse des relatives en grammaire générative depuis les années 1960 jusqu'à nos jours, et souligne le rôle fondamental du déterminant.

Dans cette troisième section toujours, trois articles reviennent sur des questions de syntaxe, branche de la linguistique chère à Françoise Dubois-Charlier. Geneviève Girard examine d'un point de vue syntactico-sémantique l'expression de la causation en français, distinguant les verbes qui fonctionnent à la fois transitivement et intransitivement et ceux nécessitant la construction FAIRE FAIRE. Laetitia Leonarduzzi et Sophie Herment se penchent sur la notion de subordination dans les interrogatives contenant une inversion et accompagnées d'un terme introducteur de subordonnées interrogatives en anglais oral. L'article de Jean-Marie Merle revient également sur la notion de subordination, et ré-analyse comme telle la subordination temporelle dite «subordination inverse» en montrant que la subordination 
sémantique et discursive n'a pas lieu de s'interpréter dans cette construction comme une inversion de la subordination syntaxique.

Trois articles, enfin, reflètent des domaines plus éloignés des préoccupations de Françoise Dubois-Charlier, mais n'en restent pas moins des travaux d'auteurs qui ont été influencés au moins dans leurs débuts par leur directrice de thèse ou de mémoire. Il s'agit tout d'abord de l'article de Lynn Blin, qui étudie une œuvre littéraire, la très courte nouvelle « How I Know What I Like » de Lydia Davis, sous le prisme linguistique afin de montrer comment l'auteure exploite la modalité, les mots de fonction et la ponctuation pour faire naître du sens au-delà du sens même des mots. L'article d' Olivier Polge, quant à lui, étudie les mécanismes de l'explicitation de l'intersubjectivité dans trois domaines différents de l'anglistique : la traduction des adverbes de l'anglais vers le français, l'évolution diachronique des verbes de volition et l'explicitation des points de vue en didactique. Enfin, l'article de Laurent Rouveyrol reprend et développe, par une analyse aussi bien qualitative que quantitative, dans une perspective logométrique, un chapitre de sa thèse dirigée par Françoise DuboisCharlier; l'article analyse le rôle du modérateur dans le débat politique au travers de deux journalistes de l'émission Question Time et montre le rôle fondamental du mode de l'opposition.

\section{L'héritage de Jean Dubois et Françoise Dubois- Charlier}

Lorsque l'on parle de Jean Dubois et Françoise Dubois-Charlier aux personnes qui les ont côtoyés de près ou de loin, les termes qui reviennent le plus souvent sont la bienveillance, l'ouverture d'esprit, le respect, la rigueur, la gentillesse, l'humilité, le talent, la générosité, sans oublier le sens de l'humour (mais aussi l'élégance). Autant de qualités humaines et intellectuelles qui font de Jean Dubois et Françoise DuboisCharlier des linguistes exceptionnels dont l'influence a été considérable et qui ont laissé une trace indélébile chez leurs disciples mais aussi chez ceux qui ont poursuivi leur carrière sur une autre voie. Ils ont su instiller chez les jeunes chercheurs la passion de la recherche. Nombreux sont ceux qui ont bénéficié dans leurs débuts de leur bienveillance et de leur confiance et qui leur doivent leur amour de la linguistique, ou qui ont simplement, à divers degrés, subi leur influence au travers de leurs ouvrages dans des domaines aussi variés que la lexicologie, la lexicographie, la syntaxe, la morphologie, l'analyse de discours, le traitement automatique du langage et la neurolinguistique. Tel est l'héritage extraordinaire qu'ils nous ont transmis sur le plan professionnel aussi bien que personnel. En témoignent les divers hommages et témoignages que le lecteur trouvera disséminés tout au long de ce numéro et particulièrement en conclusion de cet ouvrage. Nous laissons le lecteur découvrir ou redécouvrir ce grand parcours ${ }^{4}$, ce parcours hors normes, de Jean Dubois et Françoise Dubois-Charlier. 


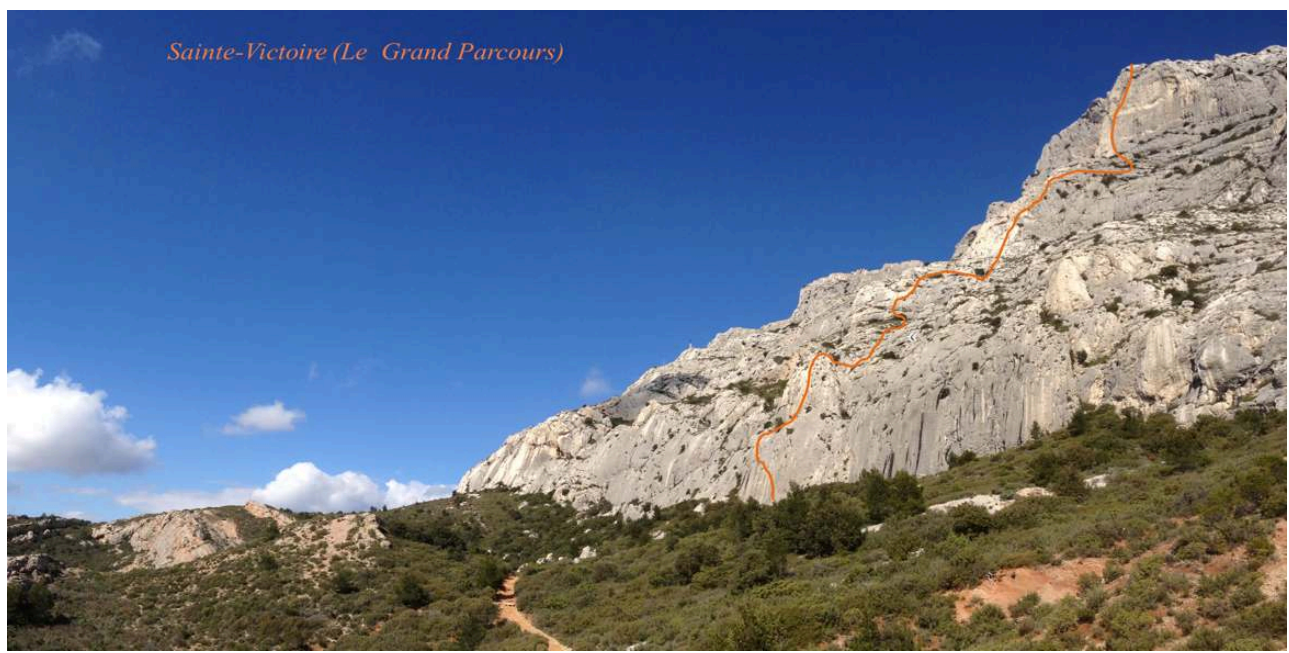

\section{NOTES}

1. Il fait suite au colloque Deux figures majeures dans l'histoire de la linguistique: Jean Dubois (1920-2015) et Françoise Dubois-Charlier (1941-2016), qui s'est tenu à Aix-en-Provence les 5 et 6 octobre 2017 dans les locaux de l'Université d'Aix Marseille, au laboratoire LPL-CNRS, et dont le but était de réunir non seulement des chercheurs mais également des personnes ayant connu Jean Dubois et Françoise Dubois-Charlier, tant d'un point de vue professionnel que personnel, et toutes unies par le même désir de leur rendre un hommage à la hauteur de ce qu'ils leur avaient apporté. Tous nos remerciements vont à Denis Le Pesant, sans qui ni le colloque d'Aix-enProvence ni ce numéro d'hommages n'auraient vu le jour. Notre reconnaissance va également à tous les contributeurs de ce numéro pour leurs réponses à nos nombreuses sollicitations.

2. Ce numéro ne comporte toutefois pas d'article spécifique sur la neurolinguistique/ psycholinguistique qui faisait partie des préoccupations de Jean Dubois et Françoise DuboisCharlier. Une présentation a été faite sur ce thème lors du colloque de 2017, que l'on peut retrouver ici: Teichmann M., Sharifzadeh S., et al. (2019). "Does surface dyslexia/dysgraphia relate to semantic deficits in the semantic variant of primary progressive aphasia?", in Neuropsychologia (Elsevier).

3. Plusieurs chercheurs de l'université de Stuttgart (Allemagne), que nous remercions pour leur participation au colloque d'Aix-en-Provence - Achim Stein, Ingrid Falk et Laura Pizzolante - ont travaillé sur le dictionnaire électronique LVF.

4. «Le grand parcours » est le nom d'une grande voie d'escalade sur la Sainte-Victoire, qui part du pied et aboutit au sommet de cette montagne (voir le tracé orange sur l'image ci-dessus). La Sainte-Victoire constituait le paysage quotidien de Jean Dubois et Françoise Dubois-Charlier lorsqu'ils résidaient à Aix-en-Provence. 


\section{AUTEUR}

\section{LAETITIA LEONARDUZZI}

Aix Marseille Université, CNRS, LPL, Aix-en-Provence, France 